\title{
ON THE SOLVABILITY OF FIRST-ORDER DISCONTINUOUS SCALAR INITIAL AND BOUNDARY VALUE PROBLEMS
}

\author{
R. P. AGARWAL ${ }^{1}$ and S. HEIKKIL $\ddot{A}^{2}$
}

(Received 27 January, 2000; revised 7 July, 2000)

\begin{abstract}
In this paper we derive extremality and comparison results for explicit and implicit initial and boundary value problems of first-order differential equations. Both the differential equations and the boundary conditions may involve discontinuities.
\end{abstract}

\section{Introduction}

Recently, the existence of Carathéodory solutions of the differential equation

$$
u^{\prime}(t)=g(t, u(t))
$$

with given initial or boundary conditions, has been proved under various kinds of hypotheses which allow $g$ to be discontinuous in both its variables (see for example $[1,2,4-14,16,18,19])$. A culmination in this research was achieved in [5], where existence of extremal solutions of the initial value problem (1.1) was proved for a large class of discontinuous functions $g$. The results of [5] were applied in [18] to prove existence results for (1.1) equipped with discontinuous functional boundary conditions.

In this paper this research is continued as follows:

(a) Existence results of $[5,18]$ are extended to the case when $u^{\prime}(t)$ is replaced in (1.1) by $d \varphi(u(t)) / d t$, where $\varphi: \mathbb{R} \rightarrow \mathbb{R}$ is an increasing homeomorphism.

(b) The $L^{1}$-boundedness of $g$ assumed in [5] is replaced by a weaker growth condition.

\footnotetext{
'Department of Mathematical Sciences, Florida Institute of Technology, Melbourne, FL 32901, USA; e-mail: agarwal@fit.edu.

${ }^{2}$ Department of Mathematical Sciences, University of Oulu, P.O. Box 3000, 90014 Oulu, Finland.

(C) Australian Mathematical Society 2003, Serial-fee code 1446-1811/03
} 
(c) When $g$ is nonnegative-valued, local conditions are introduced which allow a new type of discontinuity for $g$.

(d) Dependence of the extremal solutions on the function $g$ and on the given initial and boundary conditions is studied.

(e) The obtained results are applied to initial and boundary value problems of the differential equation $u^{\prime}(t)=q(u(t)) g(t, u(t))$.

\section{Existence and comparison results for initial value problems}

In this section we derive existence and comparison results for extremal solutions of first-order scalar initial value problems.

2.1. Hypotheses and main results Consider first the initial value problem

$$
\frac{d}{d t} \varphi(u(t))=g(t, u(t)) \quad \text { for a.e. } t \in J=\left[t_{0}, t_{1}\right], \quad u\left(t_{0}\right)=x_{0} .
$$

DEFINITION 2.1. A function $u: J \rightarrow \mathbb{R}$ is said to be a lower solution of (2.1) if $u$ belongs to the set $Y=\{u \in C(J) \mid \varphi \circ u \in A C(J)\}$ and if

$$
\frac{d}{d t} \varphi(u(t)) \leq g(t, u(t)) \text { for a.e. } t \in J, \quad u\left(t_{0}\right) \leq x_{0} .
$$

If the reversed inequalities hold, then $u \in Y$ is called an upper solution of (2.1), and a solution of (2.1) if equalities hold. If $u_{*}$ and $u^{*}$ are such solutions of (2.1) that $u_{*}(t) \leq u(t) \leq u^{*}(t)$ on $J$ for every solution $u$ of (2.1), we say that $u_{*}$ is the least solution and $u^{*}$ is the greatest solution of (2.1), and that $u_{*}$ and $u^{*}$ are the extremal solutions of (2.1).

If the functions $\varphi: \mathbb{R} \rightarrow \mathbb{R}$ and $g: J \times \mathbb{R} \rightarrow \mathbb{B}$ satisfy the following hypotheses:

$(\varphi 0) \varphi$ is an increasing homeomorphism;

(g0) for each $x \in \mathbb{B}$ the function $g(\cdot, x)$ is measurable, and

$$
\limsup _{y \dagger x} g(t, y) \leq g(t, x) \leq \liminf _{y \downarrow x} g(t, y) \quad \text { for a.e. } t \in J ;
$$

(A) (2.1) has a lower solution $\underline{u}$ and an upper solution $\bar{u}$ such that $\underline{u} \leq \bar{u}$, and $g$ is $L^{\prime}$-bounded in the set $\Omega=\{(t, x) \mid t \in J, \underline{u}(t) \leq x \leq \bar{u}(t)\}$,

we prove that there exists the least and the greatest among those solutions $u$ of (2.1) for which $\underline{u}(t) \leq u(t) \leq \bar{u}(t)$ on $J$. Replacing (A) by the following condition:

$(\mathrm{g} \varphi) \quad|g(t, x)| \leq p_{1}(t) \psi(|\varphi(x)|)$ for all $x \in \mathbb{R}$ and a.e. $t \in J$, where $p_{1} \in L_{+}^{1}(J)$, the function $\psi: \mathbb{R}_{+} \rightarrow(0, \infty)$ is increasing and $\int_{0}^{\infty} d x / \psi(x)=\infty$, 
we prove that (2.1) has extremal solutions, and that they are increasing with respect to $g$ and $x_{0}$. When $g$ is nonnegative-valued we give a localised version to condition ( $g 0$ ), which also allows downward jumps for $g(t, \cdot)$. Conditions ensuring one-sided continuities for the dependence of extremal solutions of (2.1) on $g$ and $x_{0}$ are also given.

The obtained results are then shown to hold for the IVP

$$
u^{\prime}(t)=q(u(t)) g(t, u(t)) \quad \text { a.e. in } J, \quad u\left(t_{0}\right)=x_{0},
$$

where $q: \mathbb{R} \rightarrow(0, \infty)$, if condition: $(\varphi 0)$ is replaced by the following condition.

(q0) $q$ and $1 / q$ belong to $L_{\mathrm{loc}}^{\infty}(\mathbb{R})$, and $\int_{0}^{ \pm \infty} d z / q(z)= \pm \infty$.

Finally, examples and counter-examples are given to illustrate the obtained results and the need for the given hypotheses.

REMARKS 2.1. Conditions (g0) and ( $\mathrm{q} 0$ ) allow the functions $g$ and $q$ to be discontinuous. Condition (g0) holds, for example, if $g$ is a Carathéodory function or if $g(t, x)$ is measurable in $t$ for all $x \in \mathbb{R}$ and increasing in $x$ for a.e. $t \in J$. Thus sup-measurability of $g$ is not assumed.

Condition $(\varphi 0)$ ensures only continuity of $\varphi \circ u$ when $u \in C(J)$. If $\varphi$ is locally absolutely continuous, then $\varphi^{\prime}$ is locally Lebesgue integrable, and condition $(\varphi 0)$ holds if and only if $\varphi^{\prime}$ is a.e. positive-valued and $\int_{0}^{ \pm \infty} \varphi^{\prime}(x) d x= \pm \infty$.

2.2. Preliminaries We begin with a result due to Hassan and Rzymowski, 1999, which forms a basis for the proofs of our main existence results for (2.1).

THEOREM 2.1 ([5, Theorem 3.1]). Let $f: J \times \mathbb{R} \rightarrow \mathbb{R}$ be an $L^{1}$-bounded function which satisfies the following condition:

(HR) For each $x \in \mathbb{R}$ the function $f(\cdot, x)$ is measurable, and

$$
\underset{y \nmid x}{\limsup } f(t, y) \leq f(t, x) \leq \liminf _{y \downarrow x} f(t, y) \text { for a.e. } t \in J .
$$

Then the IVP

$$
u^{\prime}(t)=f(t, u(t)) \text { for a.e. } t \in J, \quad u\left(t_{0}\right)=x_{0}
$$

has extremal solutions in $A C(J)$ for each $x_{0} \in \mathbb{R}$.

The next lemma makes condition ( $\mathrm{g} \varphi$ ) useful in proving global existence results.

LEMMA 2.1 ([12, Lemma 1.5.3]). Given $J=\left[t_{0}, t_{1}\right]$ and an increasing function $\psi: \mathbb{R}_{+} \rightarrow(0, \infty)$ for which $\int_{0}^{\infty} d x / \psi(x)=\infty$, then for all fixed $p \in L_{+}^{\prime}(J)$ and $w_{0} \in \mathbb{R}_{+}$the IVP

$$
w^{\prime}(t)=p(t) \psi(w(t)) \quad \text { for a.e. } t \in J, \quad w\left(t_{0}\right)=w_{0}
$$


has a unique solution $w \in A C(J)$. Moreover, if $v \in A C(J)$ satisfies the inequality

$$
v(t) \leq w_{0}+\int_{t_{0}}^{t} p(s) \psi(v(s)) d s, \quad t \in J
$$

then $v(t) \leq w(t)$ for all $t \in J$.

Next we shall show that $(2.1)$ can be converted to the IVP

$$
v^{\prime}(t)=g\left(t, \varphi^{-1}(v(t))\right) \text { a.e. in } J, \quad v\left(t_{0}\right)=\varphi\left(x_{0}\right)
$$

LEMMA 2.2. If condition $(\varphi 0)$ holds, then $u \in Y$ is a lower solution, an upper solution or a solution of (2.1) if and only if $v=\varphi \circ u$ is a lower solution, an upper solution or a solution of the IVP (2.3), respectively.

ProOF. If $u \in Y$ is a lower solution of (2.1), then $v=\varphi \circ u \in A C(J)$, and

$$
v^{\prime}(t)=\frac{d}{d t} \varphi(u(t)) \leq g(t, u(t))=g\left(t, \varphi^{-1}(v(t))\right) \quad \text { a.e. in } J .
$$

Moreover, since $u\left(t_{0}\right) \leq x_{0}$ and $\varphi$ is increasing, then $v\left(t_{0}\right)=\varphi\left(u\left(t_{0}\right)\right) \leq \varphi\left(x_{0}\right)$, whence $v$ is a lower solution of the IVP (2.3).

Conversely, let $v \in A C(J)$ be a lower solution of (2.3). Then $u=\varphi^{-1} \circ v \in C(J)$ by condition $(\varphi 0)$, and $\varphi \circ u=v \in A C(J)$, whence $u \in Y$, and

$$
\frac{d}{d t} \varphi(u(t))=v^{\prime}(t) \leq g\left(t, \varphi^{-1}(v(t))\right)=g(t, u(t)) \quad \text { a.e. in } J
$$

Since $v\left(t_{0}\right) \leq \varphi\left(x_{0}\right)$ and $\varphi^{-1}$ is increasing, then $u\left(t_{0}\right)=\varphi^{-1}\left(v\left(t_{0}\right)\right) \leq \varphi^{-1}\left(\varphi\left(x_{0}\right)\right)=x_{0}$. Thus $u$ is a lower solution of the IVP (2.1).

Similar reasoning shows that $u \in Y$ is a solution or an upper solution of (2.1) if and only if $v=\varphi \circ u$ is a solution or an upper solution of (2.3), respectively.

2.3. Existence and comparison results Denote by $\leq$ the pointwise ordering of $C(J)$. If $\underline{u}, \bar{u} \in C(J)$ and $\underline{u} \leq \bar{u}$, denote $[\underline{u}, \bar{u}]=\{u \in C(J) \mid \underline{u} \leq u \leq \bar{u}\}$.

We now prove our first existence and comparison result for (2.1).

THEOREM 2.2. Assume that conditions $(\varphi 0),(\mathrm{g} 0)$ and (A) hold. Then the IVP (2.1) has the least solution $u_{*}$ and the greatest solution $u^{*}$ in $[\underline{u}, \bar{u}]$. Moreover,

$$
\left\{\begin{array}{l}
u_{*}(t)=\min \left\{u_{+}(t) \mid u_{+} \text {is an upper solution of }(2.1) \text { in }[\underline{u}, \bar{u}]\right\} \\
u^{*}(t)=\max \left\{u_{-}(t) \mid u_{-} \text {is a lower solution of }(2.1) \text { in }[\underline{u}, \bar{u}]\right\}
\end{array}\right.
$$


PrOOF. Condition (A) and Lemma 2.2 imply that the functions $\underline{v}=\varphi \circ \underline{u}$ and $\bar{v}=\varphi \circ \bar{u}$ are lower and upper solutions of the IVP (2.3), and that $\underline{v} \leq \bar{v}$. If $t \in J$ and $x \in[\underline{v}(t), \bar{v}(t)]$, then $\varphi^{-1}(x) \in[\underline{u}(t), \bar{u}(t)]$. This result and condition (A) ensure the existence of an $M \in L^{1}(J)$ such that $\left|g\left(t, \varphi^{-1}(x)\right)\right| \leq M(t)$ for a.e. $t \in J$ and for all $x \in[\underline{v}(t), \bar{v}(t)]$. Thus the function $f: J \times \mathbb{R} \rightarrow \mathbb{R}$, defined by

$$
f(t, x):= \begin{cases}\underline{v}^{\prime}(t), & x<\underline{v}(t), \\ g\left(t, \varphi^{-1}(x)\right), & \underline{v}(t) \leq x \leq \bar{v}(t), \quad t \in J, \\ \bar{v}^{\prime}(t), & x>\bar{v}(t),\end{cases}
$$

is $L^{1}$-bounded. Applying conditions (g0) and $(\varphi 0)$ it is also easy to see that $f$ has the properties given in condition (HR). It then follows from Theorem 2.1 that the IVP

$$
v^{\prime}(t)=f(t, v(t)) \quad \text { for a.e. } t \in J, \quad v\left(t_{0}\right)=\varphi\left(x_{0}\right),
$$

has the least solution $v_{*}$ and the greatest solution $v^{*}$. To prove that $v_{*}$ and $v^{*}$ are extremal solutions of (2.3) in [ $\underline{v}, \bar{v}]$ we show that every solution $v$ of (2.6) belongs to the order interval $[\underline{v}, \bar{v}]$. For if $\underline{v} \notin v$, there exist $a, b \in J, a<b$, such that

$$
v(a)=\underline{v}(a) \quad \text { and } \quad v(t)<\underline{v}(t) \quad \text { on }(a, b] .
$$

Since $v$ is a solution of (2.6) with $f$ defined by (2.5), we obtain

$$
\underline{v}^{\prime}(t)-v^{\prime}(t)=\underline{v}^{\prime}(t)-f(t, v(t))=\underline{v}^{\prime}(t)-\underline{v}^{\prime}(t)=0 \quad \text { for a.e. } t \in(a, b) .
$$

Thus $\underline{v}(t)-v(t)=\underline{v}(a)-v(a)+\int_{a}^{t}\left(\underline{v}^{\prime}(s)-v^{\prime}(s)\right) d s \leq 0, t \in(a, b]$, which contradicts (2.7), and hence implies that $\underline{v} \leq v$. Similarly, it can be shown that if $v$ is a solution of (2.6), then $v \leq \bar{v}$. This and (2.5) imply that $v$ is a solution of (2.6) if and only if $v$ is a solution of (2.3) in $[\underline{v}, \bar{v}]$. This proves that $v_{*}$ and $v^{*}$ are extremal solutions of $(2.3)$ in $[\underline{v}, \bar{v}]$. Since $\varphi^{-1}$ is strictly increasing, it then follows by Lemma 2.2 that $u_{*}=\varphi^{-1} \circ v_{*}$ and $u^{*}=\varphi^{-1} \circ v^{*}$ are extremal solutions of (2.1) in $[\underline{u}, \bar{u}]$. To prove (2.4), let $u_{+}$be an upper solution of (2.1) in [ $\left.\underline{u}, \bar{u}\right]$. Replacing $\bar{u}$ by $u_{+}$in the above proof it follows that the IVP $(2.1)$ has a solution $u \in\left[\underline{u}, u_{+}\right] \subseteq[\underline{u}, \bar{u}]$. But $u_{*}$ is the least of all the solutions of $(2.1)$ in $[\underline{u}, \bar{u}]$, so that $u_{*} \leq u_{+}$. Similarly, it can be shown that if $u_{-}$is a lower solution of (2.1) in $[\underline{u}, \bar{u}]$, then $u_{-} \leq u^{*}$. Noticing also that $u_{*}$ is an upper solution and $u^{*}$ a lower solution of (2.1), we obtain (2.4).

REMARKS 2.2. In the case when

$$
g(t, x)=\left\{\begin{array}{l}
0, x>t, \\
1, x \leq t,
\end{array} \quad t \in J=[0,1], x \in \mathbb{R}, \varphi(x) \equiv x \text { and } x_{0}=0,\right.
$$


condition (g0) does not hold although for these $g, \varphi$ and $x_{0}$ the IVP (2.1) has a unique solution $u(t)=t, t \in J$ (see [5, Example 1.2]). However, by the proof of Theorem 2.2 it suffices for given $x_{0} \in \mathbb{R}$ to assume that condition (g0) holds for a.e. $t \in J$ and whenever $\underline{u}(t) \leq x \leq \bar{u}(t)$. This holds in the above case when we choose $\underline{u}(t)=0$ and $\bar{u}(t)=t, t \in J$.

As an application of Lemmas 2.1 and 2.2 and Theorem 2.2 we shall now prove the following generalisation to Theorem 2.1 .

THEOREM 2.3. Assume that the functions $\varphi: \mathbb{R} \rightarrow \mathbb{R}$ and $g: J \times \mathbb{R} \rightarrow \mathbb{R}$ satisfy conditions $(\varphi 0),(\mathrm{g} 0)$ and $(\mathrm{g} \varphi)$. Then the IVP (2.1) has for each $x_{0} \in \mathbb{R}$ the least solution $u_{*}$ and the greatest solution $u^{*}$. Moreover,

$$
\left\{\begin{array}{l}
u_{*}(t)=\min \left\{u_{+}(t) \mid u_{+} \text {is an upper solution of }(2.1)\right\} \\
u^{*}(t)=\max \left\{u_{-}(t) \mid u_{-} \text {is a lower solution of }(2.1)\right\}
\end{array}\right.
$$

Proof. Let $x_{0} \in \mathbb{R}$ be given. Choose $w_{0} \in \mathbb{R}$ so that $\left|\varphi\left(x_{0}\right)\right| \leq w_{0}$. If $v \in A C(J)$ is a solution of (2.3), it follows from condition $(\mathrm{g} \varphi)$ that

$$
\left|v^{\prime}(t)\right|=\left|g\left(t, \varphi^{-1}(v(t))\right)\right| \leq p_{1}(t) \psi(|v(t)|) \quad \text { a.e. in } J .
$$

Thus

$$
|v(t)| \leq\left|\varphi\left(x_{0}\right)\right|+\int_{t_{0}}^{t}\left|v^{\prime}(s)\right| d s \leq w_{0}+\int_{t_{0}}^{t} p_{1}(s) \psi(|v(s)|) d s
$$

for all $t \in J$. This implies by Lemma 2.1 that $|v(t)| \leq w(t)$ on $J$, where $w$ is the solution of the IVP

$$
w^{\prime}(t)=p_{1}(t) \psi(w(t)) \quad \text { a.e. in } J, \quad w\left(t_{0}\right)=w_{0}
$$

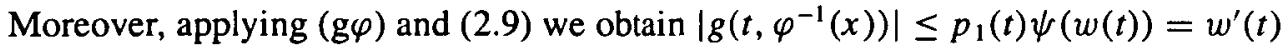
for a.e. $t \in J$, and for all $x \in[-w(t), w(t)]$. This implies that $-w$ and $w$ are lower and upper solutions of (2.3).

The above proof and Lemma 2.2 imply that $\underline{u}=\varphi^{-1} \circ(-w)$ and $\bar{u}=\varphi^{-1} \circ w$ are lower and upper solutions of the IVP (2.1), and that all the solutions of (2.1) belong to the order interval $[\underline{u}, \bar{u}]$. Moreover, if $t \in J$ and $x \in[\underline{u}(t), \bar{u}(t)]$, then $\varphi(x) \in[-w(t), w(t)]$, so that for a.e. $t \in J$ and for all $x \in[\underline{u}(t), \bar{u}(t)]$,

$$
|g(t, x)|=\mid g\left(t, \varphi^{-1}(\varphi(x)) \mid \leq p_{1}(t) \psi(w(t))=w^{\prime}(t) .\right.
$$

Thus condition (A) holds, whence the IVP(2.1) has by Theorem 2.2 extremal solutions $u_{*}$ and $u^{*}$ in $[\underline{u}, \bar{u}]$. Because all the solutions of $(2.1)$ belong to $[\underline{u}, \bar{u}]$, then $u_{*}$ and $u^{*}$ are the extremal solutions of (2.1). To prove the last assertion, let $u_{+}$be an upper 
solution of (2.1). Choose $w_{0}$ above so that $-w_{0} \leq \varphi\left(u_{+}(t)\right)$ on $J$. Then $-w \leq \varphi \circ u_{+}$, whence $\underline{u} \leq u_{+}$, so that the IVP $(2.1)$ has by Theorem 2.2 a solution $u \in\left[\underline{u}, u_{+}\right]$. But $u_{*}$ is the least of all the solutions of (2.1), so that $u_{*} \leq u_{+}$. Similarly one can show that if $u_{-}$is a lower solution of (2.1), then $u_{-} \leq u^{*}$. Since $u_{*}$ is an upper solution and $u^{*}$ a lower solution of (2.1), we obtain (2.8).

In spite of its generality, condition ( $\mathrm{g} 0$ ) does not allow the function $g(t, \cdot)$ to have jumps downwards if $t$ belongs to a complement of a fixed null-set of $J$. However, if $g$ is nonnegative-valued, ( $\mathrm{g} 0$ ) can be replaced by a localised version which allows also the jump-discontinuities mentioned above, as shown in the next theorem.

THEOREM 2.4. Assume that $\varphi: \mathbb{R} \rightarrow \mathbb{R}$ has property $(\varphi 0)$, and that $g: J \times \mathbb{R} \rightarrow \mathbb{R}$ satisfies the following conditions:

(g01) $g(\cdot, x)$ is measurable and nonnegative-valued for each $x \in \mathbb{R}$.

(g02) For each $(s, z) \in\left[t_{0}, t_{1}\right) \times \mathbb{R}$ there exist positive constants $\delta$ and $\epsilon$ such that $\limsup _{y \uparrow x} g(t, y) \leq g(t, x)$ for a.e. $t \in[s, s+\delta]$ and for all $x \in(z, z+\epsilon]$, and $g(t, x) \leq \liminf _{y \downarrow x} g(t, y)$ for a.e. $t \in[s, s+\delta]$ and for all $x \in[z, z+\epsilon)$.

(a) If condition (A) holds, then the IVP (2.1) has the least solution $u_{*}$ and the greatest solution $u^{*}$ in the order interval $[\underline{u}, \bar{u}]$, and (2.4) holds.

(b) If condition $(\mathrm{g} \varphi)$ holds, then (2.1) has for each $x_{0} \in \mathbb{R}$ extremal solutions, and (2.8) holds.

Proof. Consider first the IVP

$$
u^{\prime}(t)=g(t, u(t)) \text { for a.e. } t \in J, \quad u\left(t_{0}\right)=x_{0},
$$

where $g$ is bounded by $p_{1} \in L_{+}^{1}(J)$ and has properties (g01) and (g02), and where $x_{0} \in \mathbb{R}$ is given. Choose $\delta>0$ and $\epsilon>0$ such that $(\mathrm{g} 02)$ holds when $(s, z)=\left(t_{0}, x_{0}\right)$, and denote $J_{0}=\left[t_{0}, t_{0}+\delta\right]$. We may also assume that $\int_{J_{0}} p_{1}(t) d t<\epsilon$. Thus a function $f: J_{0} \times \mathbb{R} \rightarrow \mathbb{R}$, defined by

$$
f(t, x):=g\left(t, \max \left\{x_{0}, \min \left\{x, x_{0}+\epsilon\right\}\right), \quad t \in J_{0}, x \in \mathbb{R},\right.
$$

satisfies the hypotheses of Theorem 2.1 when $J$ is replaced by $J_{0}$, whence the IVP (2.2) has extremal solutions $u_{*}$ and $u^{*}$ on $J_{0}$. Since $g$ is nonnegative-valued, the above choices of $\delta$ and $\epsilon$ and the definitions of $J_{0}$ and $f$ ensure that (2.2) and (2.10) have the same solutions on $J_{0}$. In particular, $u_{*}$ is the least solution of $(2.10)$ on $J_{0}$. Denote

$$
t_{2}=\sup \left\{t_{3} \in J \mid(2.10) \text { has the least solution } u_{*} \text { on }\left[t_{0}, t_{3}\right]\right\} \text {. }
$$

Obviously $t_{2}=t_{1}$, for otherwise we could repeat the above reasoning when $(s, z)=$ $\left(t_{2}, u_{*}\left(t_{2}\right)\right)$, and obtain a continuation of $u_{*}$ to an interval $J_{1}=\left[t_{0}, t_{2}+\delta\right]$, which 
contradicts the choice of $t_{2}$. This proves that (2.10) has the least solution on $J$, the proof for the existence of the greatest solution being similar. The proofs of (a) and (b) are then similar to the proofs of Theorems 2.2 and 2.3.

2.4. Dependence on data As a consequence of Theorem 2.3 we obtain the following result.

Proposition 2.1. If conditions ( $\varphi 0)$, (g0) and $(\mathrm{g} \varphi)$ hold, then the IVP

$$
\frac{d}{d t} \varphi(u(t))=g(t, u(t))+h(t) \text { for a.e. } t \in J, \quad u\left(t_{0}\right)=x_{0},
$$

has for all $h \in L^{1}(J)$ and $x_{0} \in \mathbb{R}$ extremal solutions and they are increasing with respect to $x_{0}, h$ and $g$.

Proof. Given $x_{0}, \hat{x}_{0} \in \mathbb{R}, h, \hat{h} \in L^{1}(J)$ and $g, \hat{g}: J \times \mathbb{R} \rightarrow \mathbb{R}$, assume that $g$ and $\hat{g}$ have properties $(\mathrm{g} 0)$ and $(\mathrm{g} \varphi)$, and that $x_{0} \leq \hat{x}_{0}, h \leq \hat{h}$ and $g(\cdot, x) \leq \hat{g}(\cdot, x)$ for all $x \in \mathbb{R}$. The functions $(t, x) \mapsto g(t, x)+h(t)$ and $(t, x) \mapsto \hat{g}(t, x)+\hat{h}(t)$ satisfy condition (g0), and also condition $(\mathrm{g} \varphi)$ when $p_{1}$ and $\psi$ are replaced by $t \rightarrow$ $p_{1}(t)+|h(t)|+|\hat{h}(t)|$ and $z \rightarrow \psi(z)+1$, respectively. Denoting by $\hat{u}$ the least solution of the IVP

$$
\frac{d}{d t} \varphi(u(t))=\hat{g}(t, u(t))+\hat{h}(t) \text { for a.e. } t \in J, \quad u\left(t_{0}\right)=\hat{x}_{0},
$$

it follows from the above hypotheses that $\hat{u}$ is an upper solution of (2.11). This and (2.8) imply that $u_{*} \leq \hat{u}$. Similarly, it can be shown that if $\hat{u}$ is the greatest solution of (2.12), then $u^{*} \leq \hat{u}$, which concludes the proof.

Next we shall prove a result concerning right-continuity of the greatest solution of (2.1) with respect to $x_{0}$ and $g$.

PROPOSITION 2.2. Let $\varphi: \mathbb{R} \rightarrow \mathbb{R}$ satisfy condition $(\varphi 0)$, let $g_{n}: J \times \mathbb{R} \rightarrow \mathbb{R}$, $n=1,2, \ldots$, be a decreasing sequence of functions which all satisfy the hypotheses $(\mathrm{g} \varphi)$ and $(\mathrm{g} 0)$ when $g=g_{n}$, let a function $g: J \times \mathbb{R} \rightarrow \mathbb{R}$ have properties $(\mathrm{g} \varphi)$ and

(g1) for each $x \in \mathbb{R}$ the function $g(\cdot, x)$ is measurable, and

$$
\limsup _{y \uparrow x} g(t, y) \leq g(t, x)=\lim _{y \downarrow x} g(t, y) \quad \text { for a.e. } t \in J,
$$

and assume that the following condition holds:

(gn) $\lim _{n \rightarrow \infty} \sup _{x \in|a, b|}\left(g_{n}(s, x)-g(s, x)\right)=0$ for a.e. $s \in J$ and all $a, b \in \mathbb{R}, a \leq b$. 
If $\left(x_{n}\right)_{n=1}^{\infty}$ is a decreasing sequence converging to $x_{0} \in \mathbb{R}$, then the IVPs

$$
\frac{d}{d t} \varphi(u(t))=g_{n}(t, u(t)) \text { a.e. in } J, \quad u\left(t_{0}\right)=x_{n}
$$

have greatest solutions $u_{n}$, which converge uniformly on $J$ to the greatest solution $u^{*}$ of the IVP (2.1).

PROOF. The proof of Theorem 2.3 ensures that the IVP

$$
v^{\prime}(t)=g_{n}\left(t, \varphi^{-1}(v(t))\right) \text { a.e. in } J, \quad v\left(t_{0}\right)=\varphi\left(x_{n}\right)
$$

has for each $n=1,2, \ldots$ the greatest solution $v_{n}$. If $n<m$, then $x_{m} \leq x_{n}$ and $g_{m}\left(t, \varphi^{-1}(x)\right) \leq g_{n}\left(t, \varphi^{-1}(x)\right)$ in $J \times \mathbb{R}$, so that $v_{m}$ is a lower solution of (2.14). Thus $v_{m} \leq v_{n}$ by Theorem 2.3 and Lemma 2.2 , whence the sequence $\left(v_{n}\right)_{n=1}^{\infty}$ is decreasing. Denote $w_{0}=\sup \left\{\left|\varphi\left(x_{n}\right)\right| \mid n=1,2, \ldots\right\}$, and let $w$ be the solution of the IVP

$$
w^{\prime}(t)=p_{1}(t) \psi(w(t)) \text { a.e. in } J, \quad w\left(t_{0}\right)=w_{0} .
$$

Applying condition ( $\mathrm{g} \varphi$ ) we obtain for each $n=1,2, \ldots$ and for all $t \in J$,

$$
\left|v_{n}(t)\right| \leq\left|\varphi\left(x_{n}\right)\right|+\int_{t_{0}}^{t}\left|g_{n}\left(s, \varphi^{-1}\left(v_{n}(s)\right)\right)\right| d s \leq w_{0}+\int_{t_{0}}^{t} p_{1}(s) \psi\left(\mid\left(v_{n}(s) \mid\right) d s .\right.
$$

This implies by Lemma 2.1 that $\left|v_{n}(t)\right| \leq w(t)$ for each $t \in J$ and for each $n=$ $1,2, \ldots$ In particular,

$$
v_{n}(t) \in\left[-w\left(t_{1}\right), w\left(t_{1}\right)\right] \text { for all } n=1,2, \ldots \text { and } t \in J .
$$

If $t_{0} \leq a \leq b \leq t_{1}$, we have for each $n=1,2, \ldots$,

$$
\begin{aligned}
\left|v_{n}(b)-v_{n}(a)\right| & \leq \int_{a}^{b}\left|g_{n}\left(s, \mid \varphi^{-1}\left(v_{n}(s)\right)\right)\right| d s \\
& \leq \int_{a}^{b} p(s) \psi(w(s) d s=w(b)-w(a) .
\end{aligned}
$$

Thus the sequence $\left(v_{n}\right)_{n=1}^{\infty}$ is decreasing, uniformly bounded by (2.16) and equicontinuous by (2.17), whence it converges uniformly on $J$ to a function $v$ which has the property $|v(b)-v(a)| \leq|w(b)-w(a)|, a, b \in J$. In particular, $v \in A C(J)$. It follows from (2.16) when $n \rightarrow \infty$ that $v(t) \in\left[-w\left(t_{1}\right), w\left(t_{1}\right)\right]$ for all $t \in J$. Since $g_{0}(t, \cdot)$ is by $(\mathrm{g} 1)$ right-continuous for a.e. $t \in J$, since the sequence $\left(\varphi^{-1} \circ v_{n}\right)$ is decreasing and converges uniformly $\varphi^{-1} \circ v$, and since condition (gn) holds, then

$$
g_{n}\left(s, \varphi^{-1}\left(v_{n}(s)\right)\right) \rightarrow g\left(s, \varphi^{-1}(v(s))\right) \quad \text { as } n \rightarrow \infty \text { for a.e. } s \in J .
$$


Each $v_{n}$ satisfies the integral equation

$$
v_{n}(t)=\varphi\left(x_{n}\right)+\int_{t_{0}}^{t} g_{n}\left(s, \varphi^{-1}\left(v_{n}(s)\right)\right) d s, \quad t \in J
$$

Because $x_{n} \rightarrow x_{0}$ and $v_{n}(t) \rightarrow v(t)$, and since both $\varphi$ and $\varphi^{-1}$ are continuous, it follows from (2.19) when $n \rightarrow \infty$, applying also (2.18) and the dominated convergence theorem, that

$$
v(t)=\varphi\left(x_{0}\right)+\int_{t_{0}}^{t} g\left(s, \varphi^{-1}(v(s))\right) d s, \quad t \in J .
$$

This implies that $v$ is a solution of the IVP (2.3).

Denote by $\hat{v}$ the greatest solution of (2.3). Since $\hat{v}$ is a lower solution of (2.14) for each $n \in \mathbb{N}$, then $\hat{v}(t) \leq v_{n}(t), t \in J, n=1,2, \ldots$ This implies when $n \rightarrow \infty$ that $\hat{v}(t) \leq v(t)$ on $J$. The reverse inequality holds since $v$ is a solution of (2.3) and $\hat{v}$ is its greatest solution. Thus $v=\hat{v}$, that is, $v$ is the greatest solution of (2.3).

The above results and Lemma 2.2 imply that the function $u_{n}=\varphi^{-1} \circ v_{n}$ is for each $n=1,2, \ldots$ a greatest solution of the IVP (2.13), and that $\left(u_{n}\right)$ is a decreasing sequence which converges uniformly to the greatest solution of the IVP (2.1). This concludes the proof.

REMARKS 2.3. The hypothesis that the sequence $\left(x_{n}\right)_{n=1}^{\infty}$ is decreasing is essential. For instance, if $H$ is the Heaviside function: $H(z)=\left\{\begin{array}{ll}1, & z \geq 0, \\ 0, & z<0\end{array}\right.$, then the IVP

$$
u^{\prime}(t)=H(u(t)) \text { a.e. in } J, \quad u(0)=0
$$

has $u(t)=t$ as its only solution, and the IVP

$$
u_{n}^{\prime}(t)=H\left(u_{n}(t)\right) \text { a.e. in } J, \quad u_{n}(0)=-1 / n
$$

has for each $n=1,2, \ldots$ a unique solution $u_{n}(t) \equiv-1 / n$ on $J$, so that the sequence $\left(u_{n}\right)_{n=1}^{\infty}$ does not converge even pointwise to $u$ on $J$. This holds also for the solutions of the IVPs

$$
u_{n}^{\prime}(t)=H\left(u_{n}(t)-1 / n\right) \text { a.e. in } J, \quad u_{n}(0)=1 / 2 n
$$

so that the result of Proposition 2.2 does not necessarily hold if, instead of (gn), we assume that the sequence $\left(g_{n}\right)_{n=1}^{\infty}$ is increasing and converges pointwise to $g$.

Proposition 2.2 has an obvious dual for left-continuity of the least solution of $(2.1)$ with respect to $x_{0}$ and $g$. 


\subsection{A special case The IVP}

$$
u^{\prime}(t)=q(u(t)) g(t, u(t)) \text { a.e. in } J, \quad u\left(t_{0}\right)=x_{0},
$$

can be reduced to an IVP of the form $(2.1)$ if $q: \mathbb{B} \rightarrow(0, \infty)$ satisfies one of the following conditions:

(q0) $q$ and $1 / q$ belong to $L_{\text {loc }}^{\infty}(\mathbb{R})$, and $\int_{0}^{ \pm \infty} d z / q(z)= \pm \infty$;

(q1) $q \in L_{\text {loc }}^{\infty}(\mathbb{R}), 1 / q \in L_{\text {loc }}^{1}(\mathbb{B})$ and $\int_{0}^{ \pm \infty} d z / q(z)= \pm \infty$.

This is shown in the next two lemmas, the first one being an obvious consequence of the properties assumed for $q$ in conditions $(q 0)$ and $(q 1)$.

LEMMA 2.3. If (q0) or (q1) holds, then the function $\varphi: \mathbb{R} \rightarrow \mathbb{R}$, defined by

$$
\varphi(x)=\int_{0}^{x} \frac{d z}{q(z)}, \quad x \in \mathbb{R},
$$

is an increasing homeomorphism, its inverse $\varphi^{-1}$ is locally Lipschitz continuous if (q1) holds, and both $\varphi$ and $\varphi^{-1}$ are locally Lipschitz continuous if (q0) is valid. In particular, $\varphi$ satisfies condition $(\varphi 0)$.

LEMMA 2.4. If condition ( $\mathrm{q} 0)$ holds, then $u \in A C(J)$ is a lower solution, an upper solution or a solution of (2.21) if and only if $u$ is a lower solution, an upper solution or a solution of the IVP (2.1), where $\varphi: \mathbb{B} \rightarrow \mathbb{R}$ is defined by (2.22).

ProOF. Let $u$ be a solution of (2.21). Then $u \in A C(J)$, and condition (q0) ensures that $1 / q$ is measurable and locally essentially bounded. Thus an application of [17, 38.3] yields

$$
\varphi(u(t))-\varphi\left(u\left(t_{0}\right)\right)=\int_{u\left(t_{0}\right)}^{u(t)} \frac{d z}{q(z)}=\int_{t_{0}}^{t} \frac{u^{\prime}(s) d s}{q(u(s))}, \quad t \in J .
$$

This implies that $\varphi \circ u \in A C(J)$, and that

$$
\frac{d}{d t} \varphi(u(t))=\frac{d}{d t} \int_{t_{0}}^{t} \frac{u^{\prime}(s) d s}{q(u(s))}=\frac{u^{\prime}(t)}{q(u(t))}=g(t, u(t)) \text { a.e. in } J .
$$

Thus $u$ is a solution of the IVP (2.1).

Conversely, let $u \in Y$ be a solution of (2.1). Then $\varphi \circ u \in A C(J)$, and since $\varphi^{-1}$ is locally Lipschitz continuous, $u \in A C(J)$. Because

$$
\varphi(u(t))-\varphi\left(u\left(t_{0}\right)\right)=\int_{u\left(t_{0}\right)}^{u(t)} \varphi^{\prime}(z) d z=\int_{u\left(t_{0}\right)}^{u(t)} \frac{d z}{q(z)}=\int_{t_{0}}^{t} \frac{u^{\prime}(s) d s}{q(u(s))}, \quad t \in J,
$$


we obtain

$$
g(t, u(t))=\frac{d}{d t} \varphi(u(t))=\frac{d}{d t} \int_{t_{0}}^{t} \frac{u^{\prime}(s) d s}{q(u(s))}=\frac{u^{\prime}(t)}{q(u(t))} \text { a.e. in } J .
$$

Thus $u$ is a solution of the IVP (2.21).

The above proof shows that every solution of (2.21) is a solution of (2.1) and vice versa. Obvious modifications to the above proof show that problems (2.21) and (2.1) have the same upper and lower solutions.

According to Lemmas 2.3 and 2.4 the results derived for the IVPs (2.1) and (2.11) have the following consequences.

PROPOSITION 2.3. The results of Theorems 2.2 and 2.3 hold for the IVP (2.21), and the results of Proposition 2.1 hold for the IVP

$$
u^{\prime}(t)=q(u(t))(g(t, u(t))+h(t)) \text { for a.e. } t \in J, \quad u\left(t_{0}\right)=x_{0}
$$

if we replace the hypothesis $(\varphi 0)$ by condition (q0).

The next result is a consequence of Theorem 2.4 .

PROPOSITION 2.4. Assume that the functions $q: \mathbb{B} \rightarrow(0, \infty)$ and $g: J \times \mathbb{R} \rightarrow \mathbb{R}$ satisfy conditions ( $\mathrm{q} 1),(\mathrm{g} 01),(\mathrm{g} 02)$ and $(\mathrm{g} \varphi)$. Then the IVP $(2.21)$ has for each $x_{0} \in \mathbb{B}$ extremal solutions, and they are increasing with respect to $x_{0}$ and $g$.

PROOF. Because $g$ is nonnegative-valued by ( $\mathrm{g} 01$ ), then each solution of (2.21) is increasing. Hence, applying $[17,38.4]$ one can show as in the proof of Lemma 2.4 that $u \in A C(J)$ is a solution of (2.21) if and only if $u$ is a solution of the IVP (2.1), where $\varphi: \mathbb{R} \rightarrow \mathbb{R}$ is defined by (2.22). The given assumptions and Lemma 2.3 ensure that in such a case the hypotheses of Theorem 2.4 are valid, which concludes the proof.

REMARKS 2.4. The function $\varphi: \mathbb{R} \rightarrow \mathbb{R}$, defined by $\varphi(x)=|x|^{p-2} x, x \in \mathbb{R}$, satisfies condition $(\varphi 0)$ for each $p>1$. But $\varphi$ is not locally Lipschitz continuous if $p \in(1,2)$, and $\varphi^{-1}$ is not locally Lipschitz continuous if $p>2$. It then follows from Lemma 2.3 that the function $\varphi$ defined above is of the form (2.22), where $q$ has property (q0) (respectively $(q 1)$ ), only when $p=2$ (respectively $p \in(1,2]$ ). Thus problem (2.1) is more general than problem (2.21).

We can replace $\psi(|\varphi(x)|)$ by $\psi(|x|)$ in condition $(\mathrm{g} \varphi)$ if $\varphi$ is Lipschitz continuous. For if $|\varphi(x)-\varphi(y)| \leq K|x-y|, x, y \in \mathbb{R}$, for some $K>0$, then $|\varphi(x)| \leq$ $K|x|+|\varphi(0)|, x \in \mathbb{R}$, and the function $z \mapsto \psi(K x+|\varphi(0)|)$ has the properties given for $\psi$ in condition ( $g \varphi$ ). This holds for $\varphi$, defined by (2.22) if $1 / q$ is essentially bounded. 


\subsection{Examples and counter-examples}

EXAmple 2.1. Choose $J=[0,1]$ and define a function $q: \mathbb{R} \rightarrow(0, \infty)$ by

$$
q(z)=\sum_{m=1}^{\infty} \sum_{k=1}^{\infty} \frac{\left(2+\left[k^{1 / m} z\right]-k^{1 / m} z\right)}{(k m)^{2}}\left(2+\sin \left(\frac{1}{1+\left[k^{1 / m} z\right]-k^{1 / m} z}\right)\right)
$$

where $[x]$ denotes the greatest integer $\leq x$. It is easy to see that $q$ is discontinuous at $n / k^{1 / m}$ for all $n \in \mathbb{Z}, k, m=1,2, \ldots$ Moreover, $1 \leq q(z) \leq \pi^{4} / 6$ for each $z \in \mathbb{R}$, so that $q$ has property (q0).

The function

$$
g(t, x)=\sum_{m=-\infty}^{\infty} \sum_{n=1}^{\infty} \frac{f(t, x-m / n)}{2^{|m|+n}}
$$

where

$$
f(t, x)= \begin{cases}\cos (1 /(x-t))+2, & x>t \\ \chi_{U}(t), U \subset J, & x=t \\ \cos (1 /(x-t))-2, & x<t\end{cases}
$$

satisfies conditions $(\mathrm{g} 0)$ and $(\mathrm{g} \varphi)$. It then follows from Proposition 2.3 that the IVP (2.21) has extremal solutions when $q$ is given by (2.24) and $g$ by (2.25).

If $u(t) \equiv t$, then $f(\cdot, u(\cdot))$ in (2.25) is equal to the characteristic function $\chi_{U}$ of $U$, which is not measurable if $U$ is nonmeasurable. The set of all the discontinuity points of $g$ is $\{(t, t+q) \mid t \in J, q \in \mathbb{Q}\}$. This is also the set of discontinuity points of the function $g: J \times \mathbb{R} \rightarrow \mathbb{R}$, defined by (2.25), where

$$
f(t, x)= \begin{cases}(x-t) \cos (1 /(x-t)), & x>t \\ 0, & x=t \\ \cos (1 /(x-t))-2, & x<t\end{cases}
$$

which satisfies conditions (g $\varphi$ ) and (g1), assumed in Proposition 2.2.

EXAMPLE 2.2. The points

$$
c\left(n_{0}, \ldots, n_{m}\right)=1-2^{-m-1}-\sum_{k=0}^{m} 2^{-k-m-2} \prod_{j=0}^{k} 2^{-n_{j}}-2^{-2 m-2} \prod_{j=0}^{m} 2^{-n_{j}},
$$

$m, n_{0}, \ldots, n_{m} \in \mathbb{N}$, form a well-ordered set $C$ of rational numbers with $\min C=0$ and $\sup C=1$ (see [12, Example 1.1.1]). Define

$$
\begin{aligned}
f(z) & =\frac{z-c\left(n_{0}, \ldots, n_{m}\right)}{c\left(n_{0}, \ldots, n_{m}+1\right)-c\left(n_{0}, \ldots, n_{m}\right)}, \\
c\left(n_{0}, \ldots, n_{m}\right) & \leq z<c\left(n_{0}, \ldots, n_{m}+1\right), \quad m, n_{0}, \ldots, n_{m} \in \mathbb{N}
\end{aligned}
$$


and $g(t, x)=f(t+x-[t+x]), t \in J=[0,1], x \in \mathbb{R}$. It is easy to see that $g$ has properties (g01), (g02) and (g $\varphi)$, so that the IVP

$$
\frac{d}{d t}\left(|u(t)|^{p-2} u(t)\right)=f(t+u(t)-[t+u(t)]) \text { a.e. in } J, \quad x(0)=x_{0}
$$

has by Theorem 2.4 and Remark 2.3 extremal solutions when $x_{0} \in \mathbb{R}$ and $p>1$.

The IVP

$$
u^{\prime}(t)=g(t, u(t)) \text { a.e. in } J, \quad x(0)=0
$$

has no solution on $J=[0, T]$ for any $T>0$ if $g$ is one of the functions

$$
g(t, x)=\left\{\begin{array}{ll}
2, & x<t, \\
1 / 2, & x \geq t,
\end{array} \quad g(t, x)=\left\{\begin{array}{ll}
1, & x \leq 0, \\
0, & x>0,
\end{array} \quad t \in J .\right.\right.
$$

This illustrates the need of the property $\lim \sup _{y \uparrow x} g(t, y) \leq g(t, x) \leq \liminf _{y \downarrow x} g(t, y)$ for all $x \in \mathbb{R}$ and for a.e. $t \in J$, at least between assumed lower or upper solutions (see Remark 2.2) or locally (see Theorem 2.4).

The functions $\varphi: \mathbb{R} \rightarrow \mathbb{R}$ and $g: J \times \mathbb{R} \rightarrow \mathbb{R}$, defined by

$$
\varphi(x)=x^{1 / 3}, \quad g(t, x)=2 t x^{4 / 3} / 3, \quad t \in J, x \in \mathbb{R}
$$

satisfy conditions $(\varphi 0)$ and $(\mathrm{g} 0)$ but not condition $(\mathrm{g} \varphi)$. When $t_{0}=0$ and $x_{0}=1$, the IVP (2.1) can be rewritten in this case as

$$
u^{\prime}(t)=2 t u(t)^{2} \text { a.e. in } J=\left[0, t_{1}\right], \quad u(0)=1 .
$$

This IVP does not have any solution in $A C(J)$ if $t_{1} \geq 1$, since the only possible solution is $u(t)=1 /\left(1-t^{2}\right)$. Thus condition $(\mathrm{g} \varphi)$ cannot be omitted in general.

The IVP

$$
\frac{d}{d t} u^{3}(t)=3 t^{2} \cos ^{3}(\pi / t)+t \cos ^{2}(\pi / t) \sin (\pi / t) \text { a.e. in } J=[0, T], \quad u(0)=0
$$

is of the form (2.1), where

$$
\varphi(x)=x^{3}, \quad g(t, x)=3 t^{2} \cos ^{3}(\pi / t)+t \cos ^{2}(\pi / t) \sin (\pi / t), \quad t \in J, x \in \mathbb{R} .
$$

Here $\varphi$ and $g$ satisfy conditions $(\varphi 0),(g 0)$ and $(\operatorname{g} \varphi)$. It is easy to see that the only possible solution is

$$
u(t)= \begin{cases}t \cos (\pi / t), & t \in(0, T] \\ 0, & t=0\end{cases}
$$


Since $u \in C(J)$ and $\varphi \circ u=u^{3} \in A C(J)$, then $u \in Y$, whence $u$ is a solution in the sense of Definition 2.1. Since $u$ is not of bounded variation, and hence not absolutely continuous on $J=[0, T]$ for any $T>0$, this example justifies the choice of the solution set $Y$ in Definition 2.1 to be a subset of $C(J)$.

Constant multiples of the Cantor function (see [15, page 334]) are solutions to the problem

$$
\frac{d}{d t} u^{3}(t)=0 \text { a.e. in } J=[0,1], \quad u(0)=0,
$$

which then has a continuum of solutions which are continuous and monotone, having no extremal solutions. This justifies the condition $\varphi \circ u \in A C(J)$ in Definition 2.1.

Existence and comparison results derived in $[1,2,4-6,8,12,13,16,18,19]$ for initial value problems are special cases of the results derived above when $\varphi$ is the identity function.

\section{Existence results for first-order boundary value problems}

In this section we present existence and comparison results for first-order discontinuous differential equations equipped with discontinuous, implicit and functional boundary conditions. Some results of Section 2 are used in the proofs.

3.1. Hypotheses and preliminaries Consider the boundary value problem

$$
\frac{d}{d t} \varphi(u(t))=g(t, u(t)) \text { a.e. in } J=\left[t_{0}, t_{1}\right], \quad B\left(u\left(t_{0}\right), u\right)=0,
$$

where $g: J \times \mathbb{B} \rightarrow \mathbb{R}, \varphi: \mathbb{R} \rightarrow \mathbb{R}$ and $B: \mathbb{R} \times C(J) \rightarrow \mathbb{R}$. We assume that $C(J)$ is equipped with the pointwise ordering $\leq$.

DEFINITION 3.1. We say that a function $u \in C(J)$ is a lower solution of (3.1) if $\varphi \circ u \in A C(J)$ and

$$
\frac{d}{d t} \varphi(u(t)) \leq g(t, u(t)) \text { a.e. in } J, \quad B\left(u\left(t_{0}\right), u\right) \leq 0,
$$

and an upper solution of (3.1) if the reversed inequalities hold. If equalities hold, we say that $u$ is a solution of (3.1).

The following hypotheses are imposed on the functions $\varphi, g$ and $B$ :

$(\varphi 0) \varphi$ is an increasing homeomorphism;

(g0) For each $x \in \mathbb{R}$ the function $g(\cdot, x)$ is measurable and $\limsup _{y \uparrow x} g(t, y) \leq$ $g(t, x) \leq \liminf _{y \downarrow x} g(t, y)$ for a.e. $t \in J$; 
(B0) For each $x \in \mathbb{R}$ the function $B(x, \cdot)$ is decreasing and liminf $\operatorname{in}_{y x} B(y, u) \geq$ $B(x, u) \geq \limsup _{y \downarrow x} B(y, u)$ for all $u \in C(J)$.

Moreover, if

(A) (3.1) has a lower solution $\underline{u}$ and an upper solution $\bar{u}$ such that $\underline{u} \leq \bar{u}$, and $g$ is $L^{1}$-bounded in the set $\Omega=\{(t, x) \in J \times \mathbb{B} \mid \underline{u}(t) \leq x \leq \bar{u}(t)\}$,

we prove that there exists the least and the greatest among those solutions of (3.1) which belong to the order interval $[\underline{u}, \bar{u}]=\{u \in C(J) \mid \underline{u} \leq u \leq \bar{u}\}$.

If condition $(A)$ is replaced by conditions

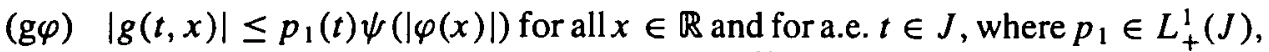
the function $\psi: \mathbb{R}_{+} \rightarrow(0, \infty)$ is increasing and $\int_{0}^{\infty} d x / \psi(x)=\infty$;

(B1) $|x-B(x, v)| \leq c|x|+d$ for all $(x, v) \in \mathbb{R} \times C(J)$, where $c \in[0,1)$ and $d \geq 0$,

we prove that (3.1) has extremal solutions, that is, the least and the greatest of all its solutions, and that they are increasing with respect to $g$ and decreasing with respect to $B$.

These results are then applied to the BVP

$$
u^{\prime}(t)=q(u(t)) g(t, u(t)) \text { a.e. in } J, \quad B\left(u\left(t_{0}\right), u\right)=0 .
$$

Existence of the extremal solutions of (3.2) is also proved under growth conditions which are different to $(\mathrm{g} \varphi)$ and (B1). Examples are given to illustrate the obtained results.

REMARK 3.1. No continuity hypotheses are imposed above on $g$ and $B$.

The following two lemmas are used in the proof of our first existence result.

LEMMA 3.1. Assume that condition (B0) holds. If $u_{1}, \ldots, u_{n}$ are solutions of the $B V P(3.1)$, then $\max \left\{u_{1}, \ldots, u_{n}\right\}$ is a lower solution of (3.1), and $\min \left\{u_{1}, \ldots, u_{n}\right\}$ is an upper solution of (3.1).

PROOF. Assume that $u_{1}, \ldots, u_{n}$ are solutions of the BVP (3.1). Since $u_{i} \in C(J)$ and $\varphi \circ u_{i} \in A C(J)$ for each $i=1, \ldots, n$, and since $\varphi$ is strictly increasing, then $u=\max \left\{u_{1}, \ldots, u_{n}\right\}$ belongs to $C(J)$ and $\varphi \circ u=\max \left\{\varphi \circ u_{1}, \ldots, \varphi \circ u_{n}\right\}$ belongs to $A C(J)$. Moreover, it is easy to show that

$$
\frac{d}{d t} \varphi(u(t))=g(t, u(t)) \text { a.e. in } J .
$$

Condition (B0) implies that if $u_{i}\left(t_{0}\right)=\max \left\{u_{1}\left(t_{0}\right), \ldots, u_{n}\left(t_{0}\right)\right\}$, then

$$
B\left(u\left(t_{0}\right), u\right)=B\left(u_{i}\left(t_{0}\right), u\right) \leq B\left(u_{i}\left(t_{0}\right), u_{i}\right)=0 .
$$


Thus $u \doteq \max \left\{u_{1}, \ldots, u_{n}\right\}$ is a lower solution of the BVP (3.1). The proof that $\min \left\{u_{1}, \ldots, u_{n}\right\}$ is an upper solution of $(3.1)$ is similar.

LEMMA 3.2. If the hypotheses ( $\mathrm{g} 0),(\varphi 0)$ and (A) are valid, and if $v$ and $w$ are lower and upper solutions of (3.1) such that $\underline{u} \leq v \leq w \leq \bar{u}$, then the IVP (2.1) has for each $x_{0} \in\left[v\left(t_{0}\right), w\left(t_{0}\right)\right]$ extremal solutions in the order interval $[v, w]$. This result holds also when condition (g0) is replaced by conditions (g01) and (g02) given in Theorem 2.4 .

PROOF. The assertions are immediate consequences of Theorems 2.2 and 2.4 .

3.2. Existence of extremal solutions of (3.1) in $[\underline{u}, \bar{u}]$ We shall first prove that the BVP (3.1) has at least one solution between the assumed lower and upper solutions $\underline{u}$ and $\bar{u}$.

PROPOSITION 3.1. If the hypotheses $(\varphi 0)$, (B0) and (A), and either (g0) or (g01) and (g02) are valid, then the BVP (3.1) has a solution in the order interval $[\underline{u}, \bar{u}]$.

PROOF. The set $X=\left\{x_{0} \in\left[\underline{u}\left(t_{0}\right), \bar{u}\left(t_{0}\right)\right] \mid\right.$ (2.1) has a solution $u \in[\underline{u}, \bar{u}]$ for which $\left.B\left(u\left(t_{0}\right), u\right) \leq 0\right\}$ is nonempty, because $(2.1)$ has for $x_{0}=\underline{u}\left(t_{0}\right)$ a solution $u$ in $[\underline{u}, \bar{u}]$ by Lemma 3.2, and condition (B0) implies that

$$
B\left(u\left(t_{0}\right), u\right)=B\left(\underline{u}\left(t_{0}\right), u\right) \leq B\left(\underline{u}\left(t_{0}\right), \underline{u}\right) \leq 0 .
$$

Denote $x^{*}=\sup X$, and let $\left(x_{n}\right)_{n=0}^{\infty}$ be an increasing sequence in $X$ which converges to $x^{*}$. The definition of $X$ allows us to choose for each $n \in \mathbb{N}$ a function $v_{n} \in[\underline{u}, \bar{u}]$ such that $\varphi \circ v_{n} \in A C(J)$ and

$$
\frac{d}{d t} \varphi\left(v_{n}(t)\right)=g\left(t, v_{n}(t)\right) \text { a.e. in } J, v_{n}\left(t_{0}\right)=x_{n} \quad \text { and } \quad B\left(v_{n}\left(t_{0}\right), v_{n}\right) \leq 0
$$

We may assume that $\left(v_{n}\right)_{n=0}^{\infty}$ is increasing, for otherwise we obtain an increasing sequence $\left(u_{n}\right)_{n=0}^{\infty}$ in $[\underline{u}, \bar{u}]$ by defining $u_{n}=\max \left\{v_{0}, \ldots, v_{n}\right\}$, and as in the proof of Lemma 3.1 it can be shown that (3.3) holds when $v_{n}$ is replaced by $u_{n}$. Condition (A) implies the existence of a function $M \in L^{1}(J)$ such that

$$
\left|\frac{d}{d t} \varphi\left(v_{n}(t)\right)\right|=\left|g\left(t, v_{n}(t)\right)\right| \leq M(t) \text { a.e. in } J
$$

whence $\left|\varphi\left(v_{n}\left(t_{3}\right)\right)-\varphi\left(v_{n}\left(t_{2}\right)\right)\right| \leq \int_{t_{2}}^{t_{3}} M(t) d t$ for all $t_{2}, t_{3} \in J, t_{2} \leq t_{3}$. Thus $\left(\varphi \circ v_{n}\right)$ is an absolutely continuous and increasing sequence in $[\varphi \circ \underline{u}, \varphi \circ \bar{u}]$. This and condition 
$(\varphi 0)$ imply that $\left(v_{n}\right)$ is an increasing sequence in $[\underline{u}, \bar{u}]$, so that it converges to a function $v: J \rightarrow \mathbb{R}$ on $J$. When $n \rightarrow \infty$ in the above inequality we obtain

$$
\left|\varphi\left(v\left(t_{3}\right)\right)-\varphi\left(v\left(t_{2}\right)\right)\right| \leq \int_{t_{2}}^{t_{3}} M(t) d t \text { for all } t_{2}, t_{3} \in J, t_{2} \leq t_{3} .
$$

This implies that $\varphi \circ v \in A C(J)$, whence $v \in C(J)$ by condition ( $\varphi 0)$. It follows from (3.3) that $\varphi\left(v_{n}\left(t_{3}\right)\right)-\varphi\left(v_{n}\left(t_{2}\right)\right)=\int_{t_{2}}^{t_{3}} g\left(t, v_{n}(t)\right) d t$ for all $n \in \mathbb{N}$ and $t_{2}, t_{3} \in J$, $t_{2} \leq t_{3}$. Allowing $n$ to tend to infinity and applying Fatou's lemma we obtain

$$
\int_{t_{2}}^{t_{3}} \frac{d}{d t} \varphi(v(t)) d t=\varphi\left(v\left(t_{3}\right)\right)-\varphi\left(v\left(t_{2}\right)\right) \leq \int_{t_{2}}^{t_{3}} \limsup _{n \rightarrow \infty} g\left(t, v_{n}(t)\right) d t
$$

for all $t_{2}, t_{3} \in J, t_{2} \leq t_{3}$. In view of this inequality and condition (g0) we get

$$
\frac{d}{d t} \varphi(v(t)) \leq \limsup _{n \rightarrow \infty} g\left(t, v_{n}(t)\right) \leq \limsup _{y \uparrow v(t)} g(t, y) \leq g(t, v(t)) \text { a.e. in } J .
$$

The last inequality of (3.3) and condition (B0) ensure that

$$
B\left(v_{n}\left(t_{0}\right), v\right) \leq B\left(v_{n}\left(t_{0}\right), v_{n}\right) \leq 0 \text { for all } n \in \mathbb{N} .
$$

These inequalities and another application of condition (B0) yields

$$
B\left(v\left(t_{0}\right), v\right) \leq \liminf _{y \uparrow v\left(t_{0}\right)} B(y, v) \leq \liminf _{n \rightarrow \infty} B\left(v_{n}\left(t_{0}\right), v\right) \leq 0,
$$

which, together with (3.4), implies that $v$ is a lower solution of (3.1).

To prove that the BVP (3.1) has a solution in the order interval $[v, \bar{u}]$, assume first that $x^{*}=v\left(t_{0}\right)=\bar{u}\left(t_{0}\right)$. Then the IVP $(2.1)$, with $x_{0}=x^{*}$, has by Lemma 3.2 a solution $u$ in $[v, \bar{u}]$. Since $u\left(t_{0}\right)=v\left(t_{0}\right)=\bar{u}\left(t_{0}\right)$ and $v \leq u \leq \bar{u}$, condition (B0) implies that

$$
0 \geq B\left(v\left(t_{0}\right), v\right) \geq B\left(u\left(t_{0}\right), u\right) \geq B\left(\bar{u}\left(t_{0}\right), \bar{u}\right) \geq 0 .
$$

Thus $B\left(u\left(t_{0}\right), u\right)=0$, and since $u$ is a solution of (2.1) when $x_{0}=x^{*}$, then $u$ is also a solution of $(3.1)$ in $[v, \bar{u}] \subset[\underline{u}, \bar{u}]$.

Assume next that $x^{*}=v\left(t_{0}\right)<\bar{u}\left(t_{0}\right)$, and choose a decreasing sequence $\left(y_{n}\right)$ from $\left[x^{*}, \bar{u}\left(t_{0}\right)\right]$ which converges to $x^{*}$. Dual arguments to those used in the construction of the sequence $\left(v_{n}\right)$ above show the existence of a decreasing sequence $\left(w_{n}\right)$ in the order interval $[v, \bar{u}]$ such that each $\varphi \circ w_{n}$ belongs to $A C(J)$ and

$$
\frac{d}{d t} \varphi\left(w_{n}(t)\right)=g\left(t, w_{n}(t)\right) \text { a.e. in } J, \quad w_{n}\left(t_{0}\right)=x_{n} \text { and } B\left(w_{n}\left(t_{0}\right), w_{n}\right) \geq 0,
$$

and which converges on $J$ to an upper solution $w$ of (3.1). Since $v\left(t_{0}\right)=w\left(t_{0}\right)$ and $v \leq w$, then replacing $\bar{u}$ by $w$ in the above reasoning when $v\left(t_{0}\right)=\bar{u}\left(t_{0}\right)$ one can prove that the $\operatorname{BVP}(3.1)$ has a solution in $[v, w] \subset[\underline{u}, \bar{u}]$. This concludes the proof. 
Now we are ready to prove the main result of this subsection.

THEOREM 3.1. If the hypotheses ( $\varphi 0)$, (B0) and (A), and either (g0) or (g01) and (g02) are valid, then the BVP (3.1) has extremal solutions $u_{*}$ and $u^{*}$ in the order interval $[\underline{u}, \bar{u}]$. Moreover,

$$
\left\{\begin{array}{l}
u_{*}(t)=\min \left\{u_{+}(t) \mid u_{+} \text {is an upper solution of }(3.1) \text { in }[\underline{u}, \bar{u}]\right\} \\
u^{*}(t)=\max \left\{u_{-}(t) \mid u_{-} \text {is a lower solution of }(3.1) \text { in }[\underline{u}, \bar{u}]\right\}
\end{array}\right.
$$

Proof. Denote $S=\{u \in C(J) \mid u$ is a solution of the BVP $(3.1)$ in $[\underline{u}, \bar{u}]\}$, and define a mapping $w: J \rightarrow \mathbb{R}$ by $w(t):=\sup _{u \in S} u(t), t \in J$. Let $D=\left\{t_{j}\right\}_{j \in \mathbb{N}}$ be a dense subset of $J$, and choose for each $j \in \mathbb{N}$ a sequence $\left(v_{k}^{j}\right)_{k=0}^{\infty}$ from the solution set $S$ such that $\lim _{k \rightarrow \infty} v_{k}^{j}\left(t_{j}\right)=w\left(t_{j}\right), j \in \mathbb{N}$. It follows from Lemma 3.1 that the functions $v_{n}: J \rightarrow \mathbb{R}, n \in \mathbb{N}$, defined by $v_{n}(t)=\max \left\{v_{k}^{j}(t) \mid j, k \in\{1, \ldots, n\}\right\}$, $t \in J$, are lower solutions of (3.1). Moreover, $\left(v_{n}\right)_{n=0}^{\infty}$ is an increasing sequence in $[\underline{u}, \bar{u}]$. It can be shown as in the proof of Proposition 3.1 that the sequence $\left(v_{n}\right)_{n=0}^{\infty}$ converges on $J$ to a lower solution $v$ of (3.1). The above construction implies also that $v \in[\underline{u}, \bar{u}]$, and that

$$
v\left(t_{j}\right)=w\left(t_{j}\right)=\sup _{u \in S} u\left(t_{j}\right) \text { for each } j \in \mathbb{N}
$$

In particular, the hypotheses of Proposition 3.1 hold when $\underline{u}$ is replaced by $v$, whence the BVP (3.1) has a solution $u^{*}$ in $[v, \bar{u}]$. Thus $u^{*} \in S$ and $v \leq u^{*}$. These relations imply by (3.6) that if $u$ is any solution of (3.1) in $[\underline{u}, \bar{u}]$, then

$$
u\left(t_{j}\right) \leq w\left(t_{j}\right)=u^{*}\left(t_{j}\right), \quad j \in \mathbb{N}
$$

Since $D=\left\{t_{j}\right\}_{j \in \mathbb{N}}$ is a dense subset of $J$, it then follows that $u(t) \leq u^{*}(t)$ on $J$. Thus $u^{*}$ is the greatest solution of the BVP (3.1) in $[\underline{u}, \bar{u}]$.

The proof that the BVP (3.1) has the least solution $u_{*}$ in $[\underline{u}, \bar{u}]$ is similar. To prove (3.5), let $u_{+}$be an upper solution of (3.1) in $[\underline{u}, \bar{u}]$. Replacing $\bar{u}$ by $u_{+}$in the above proof it follows that the BVP (3.1) has a solution $u \in\left[\underline{u}, u_{+}\right] \subseteq[\underline{u}, \bar{u}]$. But $u_{*}$ is the least of all the solutions of (3.1) in $[\underline{u}, \bar{u}]$, so that $u_{*} \leq u_{+}$. Similarly, it can be shown that if $u_{-}$is a lower solution of (3.1) in $[\underline{u}, \bar{u}]$, then $u_{-} \leq u^{*}$. Because $u_{*}$ is an upper solution and $u^{*}$ a lower solution of (3.1), we obtain (3.5).

3.3. Existence of extremal solutions of (3.1) As an application of Lemma 2.1 and Theorem 3.1 we now prove a result which guarantees the existence of least and greatest solutions among all the solutions of problem (3.1). 
THEOREM 3.2. Assume that the functions $\varphi: \mathbb{R} \rightarrow \mathbb{R}, g: J \times \mathbb{R} \rightarrow \mathbb{R}$ and $B: \mathbb{R} \times C(J) \rightarrow \mathbb{R}$ satisfy conditions $(\varphi 0),(\mathrm{g} 0),(\mathrm{g} \varphi),(\mathrm{B} 0)$ and $(\mathrm{B} 1)$. Then problem (3.1) has the least solution $u_{*}$ and the greatest solution $u^{*}$. Moreover,

$$
\left\{\begin{array}{l}
u_{*}(t)=\min \left\{u_{+}(t) \mid u_{+} \text {is an upper solution of }(3.1)\right\}, \\
u^{*}(t)=\max \left\{u_{-}(t) \mid u_{-}\right. \text {is a lower solution of (3.1)\}. }
\end{array}\right.
$$

These results hold also when condition ( $\mathrm{g} 0$ ) is replaced by conditions ( $\mathrm{g} 01)$ and (g02).

ProOF. Assume first that $u$ is a solution of (3.1). Applying (3.1) and conditions (B1) and $(\mathrm{g} \varphi)$ we obtain

$$
\left|u\left(t_{0}\right)\right|=\left|u\left(t_{0}\right)-B\left(u\left(t_{0}\right), u\right)\right| \leq c\left|u\left(t_{0}\right)\right|+d, \quad \text { that is, }\left|u\left(t_{0}\right)\right| \leq d /(1-c),
$$
and

$$
\left|\frac{d}{d t} \varphi(u(t))\right|=|g(t, u(t))| \leq p_{1}(t) \psi(|\varphi(u(t))|) \text { a.e. in } J .
$$

By choosing $w_{0} \in \mathbb{R}$ so that $-w_{0} \leq \varphi(-d /(1-c)), \varphi(d /(1-c)) \leq w_{0}$, we obtain

$$
|\varphi(u(t))| \leq\left|\varphi\left(u\left(t_{0}\right)\right)\right|+\int_{t_{0}}^{t} p_{1}(s) \psi(|\varphi(u(s))|) d s \leq w_{0}+\int_{t_{0}}^{t} p_{1}(s) \psi(\mid \varphi(u(s) \mid) d s
$$

for all $t \in J$. This implies by Lemma 2.1 that $|\varphi(u(t))| \leq w(t)$ on $J$, where $w$ is the solution of the IVP

$$
w^{\prime}(t)=p_{1}(t) \psi(w(t)), \text { a.e. in } J, \quad w\left(t_{0}\right)=w_{0} .
$$

Defining

$$
\underline{u}(t)=\varphi^{-1}(-w(t)), t \in J, \quad \text { and } \quad \bar{u}(t)=\varphi^{-1}(w(t)), t \in J,
$$

the above considerations, choice of $w_{0}$ and condition $(\varphi 0)$ imply that $u \in[\underline{u}, \bar{u}]$. Next we shall show that $\underline{u}$ and $\bar{u}$ are lower and upper solutions of (3.1). Since $w$, as a solution of (3.8), belongs to $A C(J)$, it follows from (3.9) that $\varphi \circ \underline{u}=-w$ and $\varphi \circ \bar{u}=w$ belong to $A C(J)$. Thus $\underline{u}$ and $\bar{u}$ belong to $C(J)$ by condition $(\varphi 0)$. Applying $(\mathrm{g} \varphi),(3.8)$ and (3.9) we obtain and

$$
\frac{d}{d t} \varphi(\underline{u}(t))=-w^{\prime}(t)=-p_{1}(t) \psi(w(t))=-p_{1}(t) \psi(|\varphi(\underline{u}(t))|) \leq g(t, \underline{u}(t))
$$

$$
\frac{d}{d t} \varphi(\bar{u}(t))=w^{\prime}(t)=p_{1}(t) \psi(w(t))=p_{1}(t) \psi(|\varphi(\bar{u}(t))|) \geq g(t, \bar{u}(t)),
$$

for a.e. $t \in J$. The choice of $w_{0}$ and monotonicity of $\varphi^{-1}$ imply that

$$
\underline{u}\left(t_{0}\right)=\varphi^{-1}\left(-w_{0}\right) \leq-d /(1-c), \quad d /(1-c) \leq \varphi^{-1}\left(w_{0}\right)=\bar{u}\left(t_{0}\right) .
$$


Hence

$$
\begin{aligned}
-B\left(\bar{u}\left(t_{0}\right), \bar{u}\right) & =\bar{u}\left(t_{0}\right)-B\left(\bar{u}\left(t_{0}\right), \bar{u}\right)-\bar{u}\left(t_{0}\right) \\
& \leq c\left|\bar{u}\left(t_{0}\right)\right|+d-\bar{u}\left(t_{0}\right)=c \bar{u}\left(t_{0}\right)+d-\bar{u}\left(t_{0}\right) \leq 0
\end{aligned}
$$

and

$$
\begin{aligned}
B\left(\underline{u}\left(t_{0}\right), \underline{u}\right) & =\underline{u}\left(t_{0}\right)+B\left(\underline{u}\left(t_{0}\right), \underline{u}\right)-\underline{u}\left(t_{0}\right) \\
& \leq c\left|\underline{u}\left(t_{0}\right)\right|+d+\underline{u}\left(t_{0}\right)=-c \underline{u}\left(t_{0}\right)+d+\underline{u}\left(t_{0}\right) \leq 0 .
\end{aligned}
$$

Moreover, applying $(\mathrm{g} \varphi),(3.8)$ and (3.9), we see that

$$
|g(t, x)| \leq p_{1}(t) \psi(|\varphi(x)|) \leq p_{1}(t) \psi(w(t))=w^{\prime}(t)
$$

for a.e. $t \in J$, and for all $x \in[\underline{u}(t), \bar{u}(t)]$, whence condition (A) holds.

The above proof shows that the hypotheses of Theorem 3.1 are satisfied, whence problem (3.1) has extremal solutions $u_{*}$ and $u^{*}$ in $[\underline{u}, \bar{u}]$. Because all the solutions of (3.1) belong to $[\underline{u}, \bar{u}]$, then $u_{*}$ and $u^{*}$ are the extremal solutions of (3.1). To prove (3.7), let $u_{+}$be an upper solution of (3.1). Choose $d$ in condition (B1) so that $-d /(1-c) \leq \varphi\left(u_{+}(t)\right)$ on $J$. Then $-w \leq \varphi \circ u_{+}$, whence $\underline{u} \leq u_{+}$, so that problem (3.1) has by Theorem 3.1 a solution $u \in\left[\underline{u}, u_{+}\right]$. But $u_{*}$ is the least of all the solutions of (3.1), whence $u_{*} \leq u_{+}$. Similarly, it can be shown that if $u_{-}$is a lower solution of (3.1), then $u_{-} \leq u^{*}$. Noticing also that $u_{*}$ is an upper solution and $u^{*}$ a lower solution of (3.1), we obtain (3.7).

As a consequence of Theorem 3.2 we obtain the following result.

PROPOSITION 3.2. If conditions ( $\varphi 0)$, (B0), (B1), (g0) and $(\mathrm{g} \varphi)$ hold, then the problem

$$
\frac{d}{d t} \varphi(u(t))=g(t, u(t))+h(t) \text { for a.e. } t \in J, \quad B\left(u\left(t_{0}\right), u\right)=0
$$

has for all $h \in L^{1}(J)$ extremal solutions and they are increasing with respect to $h$ and decreasing with respect to $B$.

Proof. Given $h, \hat{h} \in L^{1}(J)$ and $B, \hat{B}: J \times C(J) \rightarrow \mathbb{R}$, assume that $B$ and $\hat{B}$ have properties (B0) and (B1), and that $h \leq \hat{h}$ and $B(x, u) \geq \hat{B}(x, u)$ for all $(x, u) \in \mathbb{R} \times C(J)$. The functions $(t, x) \mapsto g(t, x)+h(t)$ and $(t, x) \mapsto \hat{g}(t, x)+\hat{h}(t)$

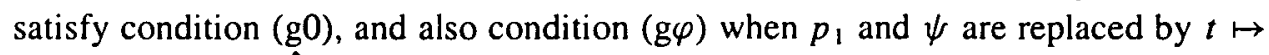
$p_{1}(t)+|h(t)|+|\hat{h}(t)|$ and $z \mapsto \psi(z)+1$, respectively. Denoting by $\hat{u}$ the least solution of the problem

$$
\cdot \frac{d}{d t} \varphi(u(t))=\hat{g}(t, u(t))+\hat{h}(t) \text { for a.e. } t \in J, \quad \hat{B}\left(u\left(t_{0}\right), u\right)=0
$$


then $\hat{u}$ is an upper solution of (3.10). This and (3.7) imply that $u_{*} \leq \hat{u}$. Similarly, it can be shown that if $\hat{u}$ is the greatest solution of (3.10), then $u^{*} \leq \hat{u}$, which concludes the proof.

EXAMPLE 3.1. Choose $J=[0,1]$ and consider the problem

$$
\left\{\begin{array}{l}
u^{\prime}(t)=H(u(t)-4 t)+\frac{[u(t)]}{1+|[u(t)]|} \text { a.e. in } J \\
u(0)=\frac{[u(0)]}{1+|[u(0)]|}+\frac{2\left[\int_{0}^{1} u(s) d s\right]}{1+\left|\left[\int_{0}^{1} u(s) d s\right]\right|}
\end{array}\right.
$$

where $H$ is the Heaviside function and $[x]$ denotes the greatest integer less than or equal to $x$. Problem (3.11) is of the form (3.1) with

$$
\begin{cases}g(t, x)=H(x-4 t)+\frac{[x]}{1+|[x]|}, & t \in J, x \in \mathbb{R}, \\ B(x, u)=x-\frac{[x]}{1+|[x]|}-\frac{2\left[\int_{0}^{1} u(t) d t\right]}{1+\left|\left[\int_{0}^{1} u(t) d t\right]\right|}, & x \in \mathbb{R}, u \in C(J) .\end{cases}
$$

It is easy to see that the hypotheses of Theorem 3.2 hold, whence problem (3.11) has the least solution $u_{*}$ and the greatest solution $u^{*}$. These extremal solutions can be determined by using numerical integration methods and inference. Denoting by $\chi_{W}$ the characteristic function of $W \subset \mathbb{R}$, we get the following representations for $u_{*}$ and $u^{*}$ :

$$
\left\{\begin{aligned}
u_{*}(t)= & (-9 / 4-3 t / 4) \chi_{[0,1]}(t), \quad t \in J \\
u^{*}(t)= & (13 / 6+5 t / 3) \chi_{[0,1 / 2]}(t)+(17 / 8+7 t / 4) \chi_{[1 / 2,17 / 18]}(t) \\
& +(221 / 72+3 t / 4) \chi_{[17 / 18,1]}(t), \quad t \in J
\end{aligned}\right.
$$

The function $u(t) \equiv 0$ is also a solution of (3.11).

3.4. Special cases In this subsection we shall consider solvability of the BVP (3.2) where $g: J \times \mathbb{R} \rightarrow \mathbb{R}, q: \mathbb{R} \rightarrow(0, \infty)$ and $B: \mathbb{R} \times C(J) \rightarrow \mathbb{R}$. A function $u \in A C(J)$ is said to be a lower solution of the BVP (3.2) if

$$
u^{\prime}(t) \leq q(u(t)) g(t, u(t)) \text { for a.e. } t \in J, \text { and } B\left(u\left(t_{0}\right), u\right) \leq 0
$$

and an upper solution if the reversed inequalities hold. If equalities hold, we say that $u$ is a solution of the BVP (3.2).

Lemma 2.3 and the proof of Lemma 2.4 imply the following result. 
LEMMA 3.3. Assume that $q: \mathbb{B} \rightarrow(0, \infty)$ satisfies the following condition:

(q0) $q$ and $1 / q$ belong to $L_{\mathrm{loc}}^{\infty}(\mathbb{R})$ and $\int_{0}^{ \pm \infty} d z / q(z)= \pm \infty$.

Then $u \in A C(J)$ is a lower solution, an upper solution or a solution of (3.2) if and only if $u$ is a lower solution, an upper solution or a solution of the BVP (3.1), respectively, where $\varphi: \mathbb{R} \rightarrow \mathbb{B}$ is defined by

$$
\varphi(x)=\int_{0}^{x} \frac{d z}{q(z)}, \quad x \in \mathbb{R} .
$$

Moreover, $\varphi$ satisfies condition $(\varphi 0)$.

In view of Lemma 3.3 we obtain the following result.

PROPOSITION 3.3. The results of Theorems 3.1 and 3.2 hold for problem (3.2) if condition $(\varphi 0)$ is replaced by condition $(\mathrm{q} 0)$.

The next result gives another sufficient condition for the existence of extremal solutions of the BVP (3.2).

THEOREM 3.3. Assume that functions $g, B$ and $q$ have properties (g0), (B0) and

(g2) $|g(t, x)| \leq M(t)+p_{1}(t)|x|$ for a.e. $t \in J$ and all $x \in \mathbb{R}$, where $p_{1}, M \in$ $L_{+}^{1}(J)$;

(q2) $q$ is measurable and essentially bounded and $\frac{1}{q}$ is locally essentially bounded. Assume also the existence of such constants $a>0$ and $b, c \geq 0$ and $a$ bounded and nonnegative linear functional I on $C(J)$ such that

(B2) $|B(x, u)-a x+b I(u)| \leq c$ for all $x \in \mathbb{R}$ and $u \in C(J)$;

(A1) $a>b I\left(e^{P(\cdot)}\right)$, where $P(t)=\int_{t_{0}}^{t}\|q\|_{\infty} p_{1}(s) d s, t \in J$.

Then the BVP (3.2) has extremal solutions, and all the solutions of (3.2) lie within the order interval $[-w, w]$, where

$$
\begin{aligned}
w(t)= & e^{P(t)} \frac{c+b I\left(\tau \mapsto \int_{t_{0}}^{\tau} e^{P(\tau)-P(s)}\|q\|_{\infty} M(s) d s\right)}{a-b I\left(e^{P(\cdot)}\right)} \\
& +\int_{t_{0}}^{t} e^{P(t)-P(s)}\|q\|_{\infty} M(s) d s .
\end{aligned}
$$

ProOF. It follows from Lemma 3.3 that we can replace $q(x)$ by $\min \left\{q(x),\|q\|_{\infty}\right\}$, and hence assume that $q(x) \leq\|q\|_{\infty}$ for all $x \in \mathbb{R}$. It is elementary to verify that $w$, given by (3.13), is a unique solution of the BVP

$$
w^{\prime}(t)=\|q\|_{\infty}\left(M(t)+p_{1}(t) w(t)\right) \text { a.e. in } J, \quad a w\left(t_{0}\right)-b l(w)=c .
$$


Applying conditions (q2) and (g2) we obtain, for a.e. $t \in J$,

$$
w^{\prime}(t)=\|q\|_{\infty}\left(M(t)+p_{1}(t) w(t)\right) \geq q(w(t)) g(t, w(t))
$$

and

$$
-w^{\prime}(t)=\|q\|_{\infty}\left(-M(t)-p_{1}(t) w(t)\right) \leq q(-w(t)) g(t,-w(t)) .
$$

The boundary condition of (3.14) and condition (B2) imply that

$$
B\left(w\left(t_{0}\right), w\right)=B\left(w\left(t_{0}\right), w\right)-a w\left(t_{0}\right)+b l(w)+c \geq 0
$$

and

$$
B\left(-w\left(t_{0}\right),-w\right)=B\left(-w\left(t_{0}\right),-w\right)-a\left(-w\left(t_{0}\right)\right)+b I(-w)-c \leq 0 .
$$

Thus $\underline{u}=-w$ is a lower solution and $\bar{u}=w$ is an upper solution of (3.2). Moreover $|g(t, u)| \leq M(t)+p_{1}(t) w(t)$ for a.e. $t \in J$ and for all $u \in[-w(t), w(t)]$, so that $g$ is $L^{\prime}$-bounded in $\Omega$. Thus condition (A) holds when $\underline{u}=-w$ and $\bar{u}=w$. Since conditions ( $\mathrm{g} 0$ ) and $(\mathrm{B} 0)$ are assumed to hold, and since (q2) implies the validity of condition ( $\mathrm{q} 0$ ), then the BVP (3.2) has by Proposition 3.3 extremal solutions $u_{*}$ and $u^{*}$ in $[-w, w]$.

If $u$ is a solution of (3.2), it follows from (3.2) and (g $\varphi$ ) that

$$
u^{\prime}(s)-\|q\|_{\infty} p_{1}(s) u(s) \leq\|q\|_{\infty} M(s) \text { a.e. in } J .
$$

Multiplying both sides of (3.15) by $e^{-P(s)}$ and integrating from $t_{0}$ to $t$, we obtain

$$
u(t) \leq e^{P(t)} u\left(t_{0}\right)+\int_{t_{0}}^{t} e^{P(t)-P(s)}\|q\|_{\infty} M(s) d s, \quad t \in J .
$$

In view of condition (B2) and the boundary condition of (3.2), we have

$$
a u\left(t_{0}\right)-b I(u)=a u\left(t_{0}\right)-b I(u)-B\left(u\left(t_{0}\right), u\right) \leq c .
$$

This and (3.16) imply that

$$
\begin{aligned}
c & \geq a u\left(t_{1}\right)-b l(u) \\
& \geq a u\left(t_{0}\right)-b l\left(u\left(t_{0}\right) e^{P(\cdot)}\right)-b l\left(\tau \mapsto \int_{t_{0}}^{\tau} e^{P(\tau)-P(s)}\|q\|_{\infty} M(s) d s\right),
\end{aligned}
$$

so that

$$
u\left(t_{0}\right) \leq \frac{c+b I\left(\tau \mapsto \int_{t_{0}}^{\tau} e^{P(\tau)-P(s)}\|q\|_{\infty} M(s) d s\right)}{a-b I\left(e^{P(\cdot)}\right)} .
$$

It then follows from (3.13), (3.16) and (3.17) that $u(t) \leq w(t)$ for each $t \in J$, that is, $u \leq w$. Similarly one can show that $-w \leq u$, so that $u \in[-w, w]$.

The above proof shows that all the solutions of (3.2) belong to the order interval $[-w, w]$, whence $u_{*}$ and $u^{*}$ are least and greatest of all the solutions of (3.2). 
In the case when $B(x, u)=a x-b u\left(t_{1}\right)-c$ and $I u=u\left(t_{1}\right)$, we get the following consequence of Theorem 3.3.

PROPOSITION 3.4. If the functions $g$ and $q$ have properties (g0), (g2) and (q2), and if positive constants $a$ and $b$ satisfy $\int_{t_{0}}^{t_{1}} p_{1}(s) d s<\left(1 /\|q\|_{\infty}\right) \ln (a / b)$, then the BVP

$$
u^{\prime}(t)=q(u(t)) g(t, u(t)) \text { a.e. in } J=\left[t_{0}, t_{1}\right], \quad a u\left(t_{0}\right)-b u\left(t_{1}\right)=c,
$$

has for each $c \in \mathbb{R}$ extremal solutions.

EXAMPLE 3.2. Let the functions $q: \mathbb{R} \rightarrow \mathbb{B}$ and $g: J \times \mathbb{R} \rightarrow \mathbb{R}, J=[0,1]$, be defined by (2.24) and (2.25). It follows from Example 2.1 that $q$ and $g$ satisfy conditions (q2), (g0) and (g2) when $p_{1}(t) \equiv 0$ and $M(t) \equiv 9$.

The function

$$
B(x, u)=2 x-\int_{J} u(t) d t+\sum_{m=-\infty}^{\infty} \sum_{n=1}^{\infty} \frac{\mu(x-m / n, u)}{2^{|m|+n}} \frac{1-\left[\int_{J} u(t) d t\right]}{1+\left|\left[\int_{J} u(t) d t\right]\right|},
$$

where

$$
\mu(x)= \begin{cases}\cos (1 / x)-2, & x>0 \\ 0, & x=0 \\ \cos (1 / x)+2, & x<0\end{cases}
$$

satisfies conditions (B0) and (B2) when $a=2, b=1, c=9$ and $I(u)=\int_{J} u(t) d t$. Also condition (A1) holds. Thus the BVP (3.2) has for these functions $q, g$ and $B$ extremal solutions by Theorem 3.3.

REMARKS 3.2. The results of this section generalise those derived in $[11,12,14]$ for periodic boundary value problems, in $[7,10]$ for problems with nonlinear boundary conditions, and in $[9,18]$ for problems with functional boundary conditions when $\varphi(x) \equiv x$, except that the lower and upper solutions are of a more general type in [18] than in Definition 3.1.

\section{References}

[1] D. C. Biles, "Continuous dependence of nonmonotonic discontinuous differential equations", Trans. Amer. Math. Soc. 339 (1993) 507-524.

[2] D. C. Biles, "Existence of solutions for discontinuous differential equations", Diff. Int. Eqns. 8 (1995) 1525-1532.

[3] C. Carathéodory, Vorlesungen über reelle Funktionen (Chelsea, New York, 1948). 
[4] S. Carl, S. Heikkilä and M. Kumpulainen, "On solvability of first-order discontinuous scalar differential equations", Nonlinear Times and Digest 2 (1995) 11-24.

[5] E. R. Hassan and W. Rzymowski, "Extremal solutions of a discontinuous scalar differential equation", Nonlinear Analysis 37 (1999) 997-1017.

[6] S. Heikkilä, On first-order discontinuous scalar differential equations, Pitman Research Notes in Mathematics Series 324 (Longman, 1995) 148-155.

[7] S. Heikkilä, "Notes on first-order discontinuous differential equations with nonlinear boundary conditions", Proc. Dynamic Sys. Appl. 2 (1996) 255-260.

[8] S. Heikkilä, "On discontinuously perturbed Carathéodory type differential equations", Nonlinear Anal. 26 (1996) 785-797.

[9] S. Heikkilä, "On first-order discontinuous differential equations with functional boundary conditions", Adv. in Nonlinear Dynamics 5 (1997) 273-281.

[10] S. Heikkilä and A. Cabada, "On first-order discontinuous differential equations with nonlinear boundary conditions", Nonlinear World 3 (1996) 487-503.

[11] S. Heikkilä and V. Lakshmikantham, "Extension of the method of upper and lower solutions for discontinuous differential equations", Diff. Eqns. and Dyn. Syst. 1 (1993) 73-86.

[12] S. Heikkilä and V. Lakshmikantham, Monotone iterative techniques for discontinuous nonlinear differential equations (Marcel Dekker, New York, 1994).

[13] S. Heikkilä and V. Lakshmikantham, "A unified theory of first-order discontinuous scalar differential equations", Nonlinear Anal. 26 (1996) 785-797.

[14] S. Heikkilä, V. Lakshmikantham and S. Leela, "Applications of monotone techniques to differential equations with discontinuous right hand side", Diff. Int. Egns. 1 (1988) 287-297.

[15] A. N. Kolmogorov and S. V. Fomin, Introductory real analysis (Prentice-Hall, Englewood Cliffs, NJ, 1970).

[16] M. Kumpulainen, "On extremal and unique solutions of discontinuous ordinary differential equations and finite systems of ordinary differential equations", Dissertation, Univ. of Oulu, Dept. of Math. Sci., 1996.

[17] E. J. McShane, Integration (Princeton Univ. Press, Princeton, NJ, 1974).

[18] R. L. Pouso, "Upper and lower solutions for first-order discontinuous ordinary differential equations", J. Math. Anal. Appl. 244 (2000) 466-482.

[19] W. Rzymowski and D. Walachowski, "One-dimensional differential equation under weak assumptions", J. Math. Anal. Appl. 198 (1996) 657-670. 